\title{
Learner-centered Design of a Web-based Teaching Tool for Circuit Analysis with Embedded Assessment Features
}

\section{Dr. Fred W. DePiero, California Polytechnic State University, San Luis Obispo}

Dr. Fred DePiero received his B.S. and M.S. degrees in Electrical Engineering from Michigan State University in 1985 and 1987. He then worked as a Development Associate at Oak Ridge National Laboratory until 1993. While there he was involved in a variety of real-time image processing projects and several laser-based ranging systems. Fred began working on his Ph.D. at the University of Tennessee while still at ORNL, and completed it in May 1996. Fred joined the faculty at CalPoly in September of 1996. He is presently serving as the Associate Dean for Student Success in the College of Engineering.

\section{Dr. Bridget Benson, California Polytechnic State University, San Luis Obispo}

Bridget Benson received a Bachelor's degree in Computer Engineering at California Polytechnic State University San Luis Obipso in 2005, a Master's degree in Electrical and Computer Engineering at the University of California Santa Barbara in 2007 and a PhD degree in the Computer Science and Engineering at the University of California San Diego in 2010. She is currently an Assistant Professor in the Electrical Engineering Department at California Polytechnic State University San Luis Obipso. Her research interests span engineering education, embedded systems, and ecological monitoring.

\section{Mr. K. Clay McKell, California Polytechnic State University, San Luis Obispo}

Clay McKell earned his B.S. degree, summa cum laude, in mechanical engineering from UCLA in 2006. He earned his M.S. degree in mechanical engineering from UCLA in 2007. He is currently pursuing a Ph.D. in electrical engineering from the University of Hawaii at Manoa and teaching as a lecturer in the Electrical Engineering Department at California Polytechnic State University, San Luis Obispo. His research interests include distributed control of multi-agent networks as well as STEM education practices that foster diversity and equity. 


\title{
Learner-Centered Design of a Web-Based Teaching Tool for Circuit Analysis With Embedded Assessment Features
}

\begin{abstract}
CATE, the Circuit Analysis Tool for Education, is a web-based teaching and learning system for linear circuit analysis. It supports active, assisted and passive learning modes. Students can tackle a particular circuit analysis problem using different learning modes and different techniques. CATE provides a learner-centered environment where the path through examples and problems is driven by a student's navigational choices. This allows students to easily transition between active, assisted and passive learning.

The purpose of assisted learning is to promote the development of abilities that are key building blocks for circuit analysis. These key abilities include the formation of KCL and KVL equations for nodal and mesh analysis. The goal of assisted learning is to lessen the frustration for students who might otherwise only have access to the final answers associated with a circuit analysis problem.
\end{abstract}

New features of CATE include the assisted learning mode, as well as the ability to transition between different learning modes for the same circuit. Another new feature is a circuit generation algorithm that can produce billions of different AC circuit topologies. CATE can now also generate exam questions with solutions.

Past student satisfaction surveys indicated a generally positive attitude towards CATE and its benefit to learning. To better examine the impact of CATE on student learning we have developed new embedded assessment tools that track students' activity longitudinally. The resolution of these data can be adjusted from a finer detail with individual clicks to more coarse versions describing runs of activity of similar type. Data sets can be augmented by uploading external assessments such as quiz grades, or by using quiz questions generated internally by CATE. Longitudinal activity and assessment data are recorded temporally in a database to form an integrated picture of student use and ability. We have also developed a feedback mechanism for an instructor that indicates which learning objectives are being investigated by students and at what difficulty level. These data describe activity in a cross-sectional fashion for a student population.

To be clear, CATE provides functionality that is different from SPICE. Each does provide values for voltages and currents in a circuit. However SPICE does not present intermediate equations associated with various types of analyses. Furthermore CATE helps address some common misconceptions such as the identification of series and parallel elements. It also provides an environment that allows students to readily build on their prior abilities by scaffolding.

CATE is a free web resource that works in a variety of browsers and is presented without any ads. Visit YourLearningCoach.com. 


\section{Introduction}

Our goal for CATE (the Circuit Analysis Tool for Education) is to supplement other learning resources, for a basic circuits course. CATE generates circuits and analyzes them in a step-by-step manner, presenting each equation alongside highlighted circuit elements. This stepwise presentation mimics how a student performs a circuit analysis. Mesh, nodal and analysis by equivalent circuits are all implemented within the CATE system. Students can select the complexity of generated circuits (referred to as a 'difficulty level'). See Figure 1. CATE also provides features useful to instructors, such as exam generation. Studies have shown that students do better when both traditional and on-line resources are available, as opposed to just one or the other [16]. CATE is intended to provide this type of benefit by complimenting a traditional course.
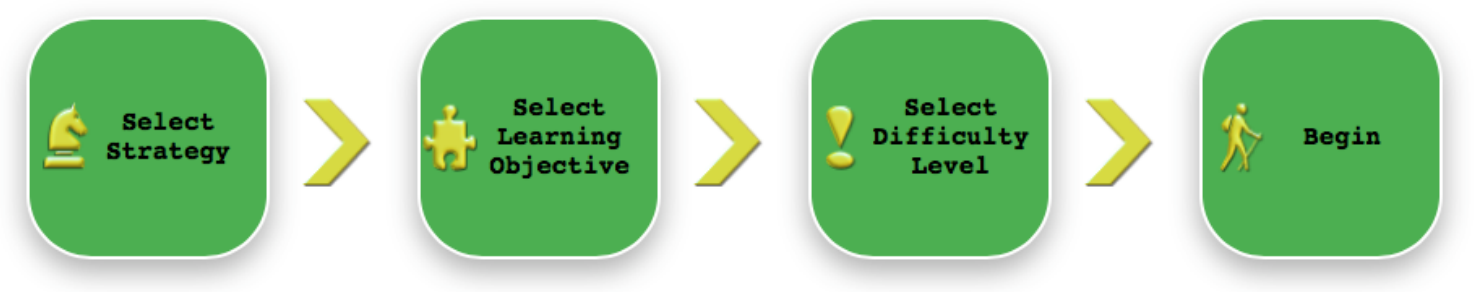

Figure 1. Students are prompted to choose their strategy (passive, assisted or active learning), then to choose a learning objective and difficulty level.

The functionality of CATE is distinct from tools such as LTSpice [17] or Circuits.IO [18]. These other tools provide final results from a circuit simulation, but do not address developmental steps in the analysis. The strength of these other tools is in yielding a final analysis, not in educating a student. CATE presents circuit equations in a step-by-step fashion (e.g. mesh or nodal), then simplifies and solves the equations to find final values for voltages and currents. The final results from CATE are just like SPICE but the intermediate steps are distinct. In this most recent version of CATE we also provide an assisted learning mode. This is intended to help students develop key abilities associated with mesh and nodal analysis. Rather then just show students the KCL and KVL equations, as in passive learning, assisted learning offers the student a chance to form these equations. Feedback is provided to the student as the terms are formed. The goal of assisted learning is to lessen the frustration for students who might otherwise only have access to the final answers associated with a circuit analysis problem. Hence learning circuit analysis with CATE is different from using a tool such as SPICE that only provides final results.

Initial evaluation of students' interest and benefits of CATE appeared positive [15]. More recently we taught a circuits course and offered students the chance to use either CATE or 'Mastering' by Pearson. Students liked the instant feedback when solving circuit problems (available in either system). However they thought the hints provided by Mastering (in the case of wrong answers) were not useful. Students also did not appreciate having to pay to access the Mastering system. 
In an effort to assess the impact of CATE on student learning, we recently examined student grades in subsequent courses. We examined grades for students in a section of a course that encouraged the use of CATE versus sections not involving CATE. To date we have not been able to demonstrate consistently better results when using CATE, in terms of average course grades. We need an mechanism that provides finer detail and have now developed an embedded assessment system that tracks student usage longitudinally. Student activity is recorded in a database and can be presented at varied resolutions. The higher resolution versions describe all the details of student activity click by click. Medium resolution versions highlight significant activity such as selection of particular learning objectives and active versus passive learning modes. Coarse presentations of the data omit the temporal dimension and just include time on task. We use the tracking data to also generate cross-sectional views of student activity.

\section{Learner-Centered Design of the CATE Environment}

CATE has been designed with a variety of features that encourage both development and retention of students' abilities. Students select learning objectives and difficulty levels as shown in Figure 1. They also choose a strategy for studying that includes passive, active or assisted learning.

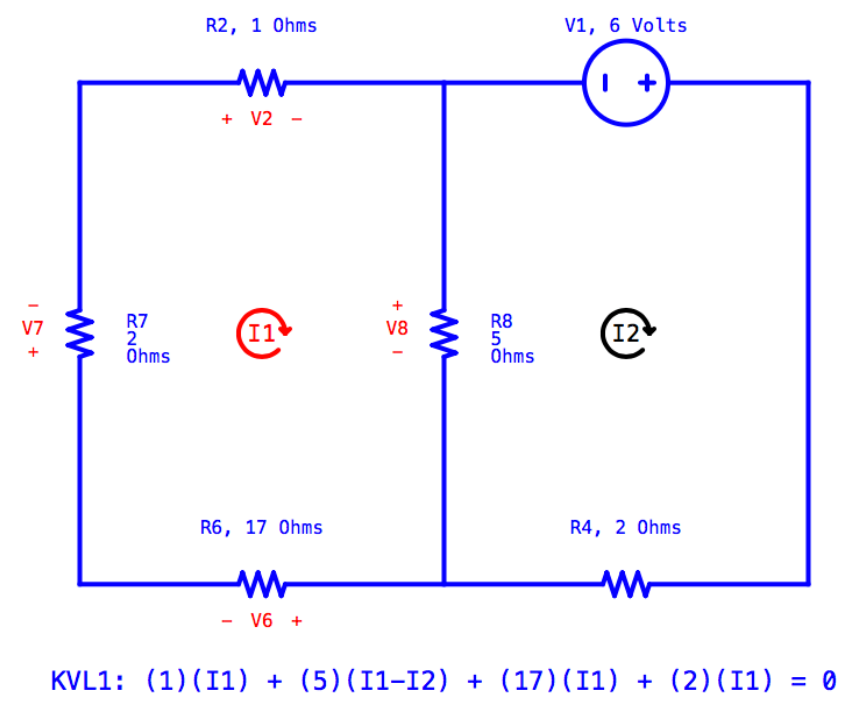

Fig 2. In passive mode CATE presents equations that parallel the steps of a student's efforts, in this case using mesh analysis.

Passive learning takes a student through an analysis in a stepwise fashion. CATE presents KVL or KCL equations as appropriate (for mesh or nodal methods). See Figure 2. Students can also select the equivalent circuit method of analysis. It performs series and parallel reductions, displaying each new version of the circuit through the analysis. 


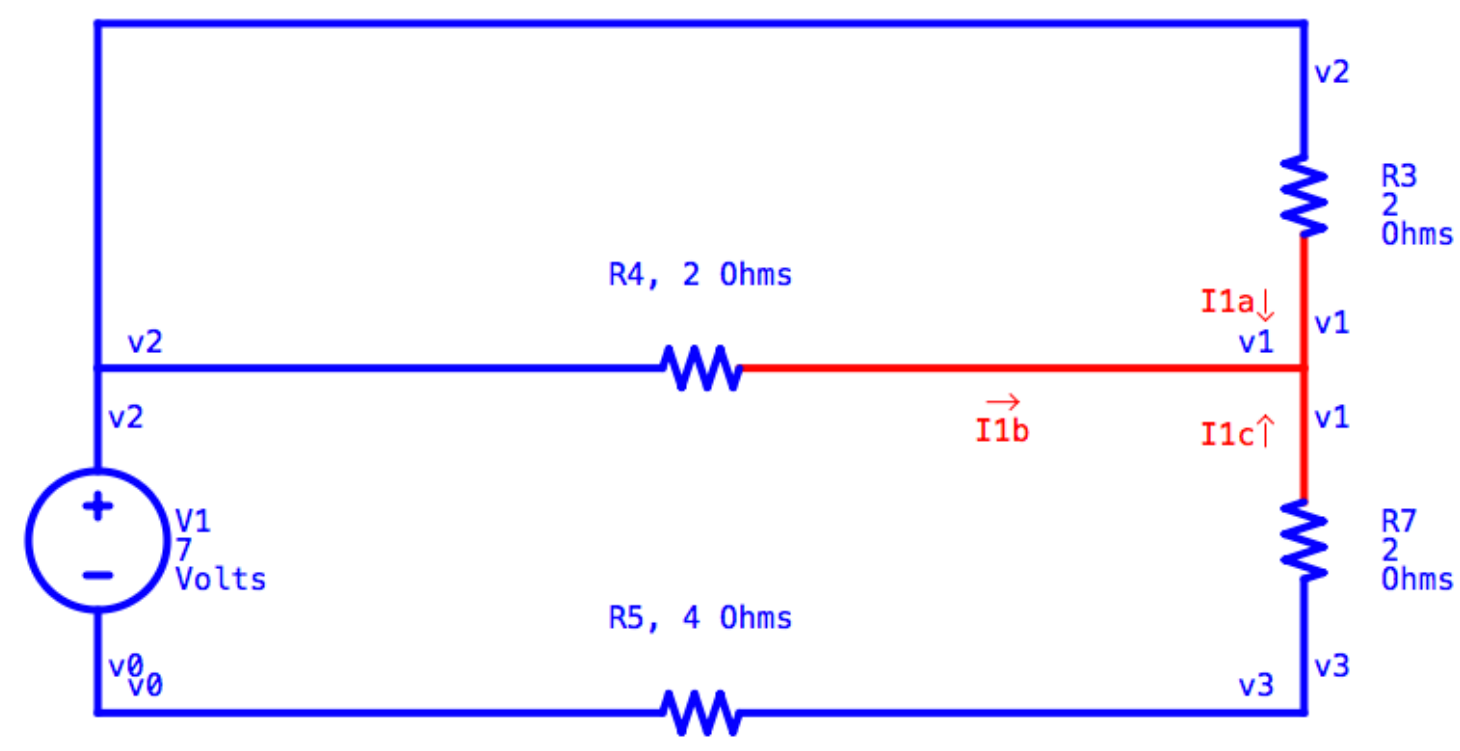

Your Eq: (1/R4) (v2-v1) + (1/R3) (v2-v1)

Form KCL equation for node 1 using terms in the form (1/Rn)(vi-vj)

Your eq has: Some unknowns present. Missing " $="$.

Fig 3. In assisted mode the student selects from an alphabet of given symbols to form equations term by term. CATE recognizes algebraically consistent equations and provides hints to the student.

Assisted learning is similar to the passive mode except that before simply presenting a KVL or KCL equation, students are asked to define it. Students manipulate an alphabet of symbols that form building blocks for a given equation. See Figure 3. Feedback helps to guide the student. The assisted learning mode was inspired by the prior work of Skromme [10-12], who showed that this style of system could accelerate student learning.

CATE's active learning mode provides a no-stakes self-testing environment encourages students to try varied example problems. See Figure 4. A subsequent review of solution details (provided by CATE) is also available to illustrate solution details, if desired by a student.

CATE's active learning mode and quizzing features are intended to both build student confidence as they verify their abilities. It also provides a check on their level of mastery, as they realize what they don't know. This follows guidance from Arnold and McDermott [2] established that rereading without self-testing can lead 
to over confidence regarding perceived mastery. Furthermore Brown [3] suggests that attempting to solve a problem and failing is better than no attempt at all. CATE provides a safe environment for failing, with no consequences to a course grade. Also CATE can generate billions of circuit topologies (for AC circuits with difficulty level 3). Work by Roediger and Karpicke [1] demonstrates that practicing retrieval promotes retention of learned abilities better than reviewing original sources. This is known as the testing effect or the retrieval-practice effect. Based on these prior studies we anticipate that CATE's large number of examples and self-testing mechanisms will promote both confidence and long-term retention for students.

What is the node voltage, $v 2$ ? Note that $v 0$ is grounded.

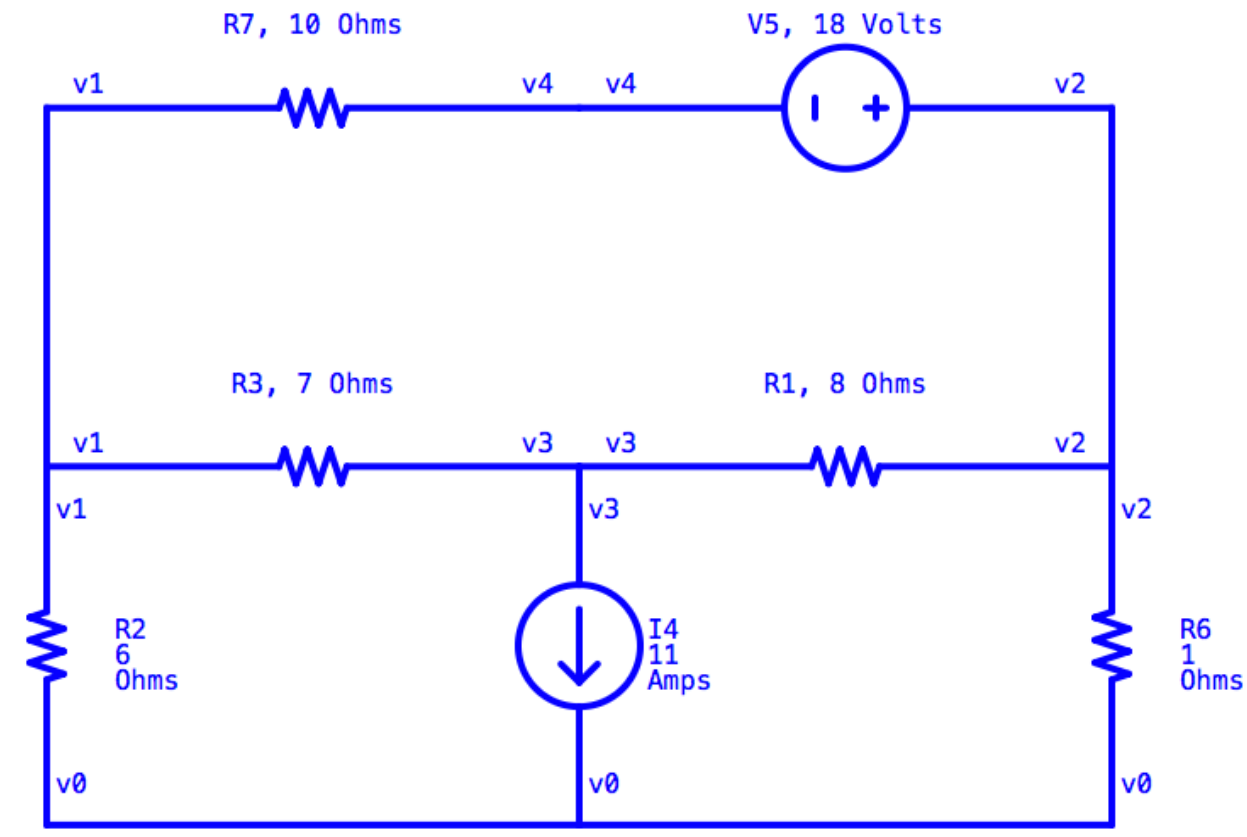

Answer in Volts

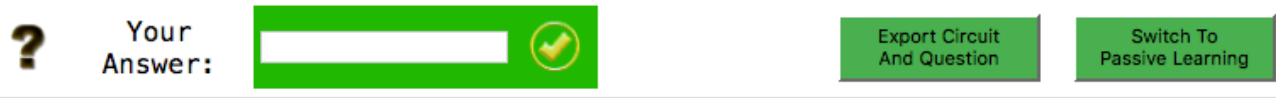

Fig 4. In active mode CATE offers no-stakes questions with automatic grading and yes/no feedback. 


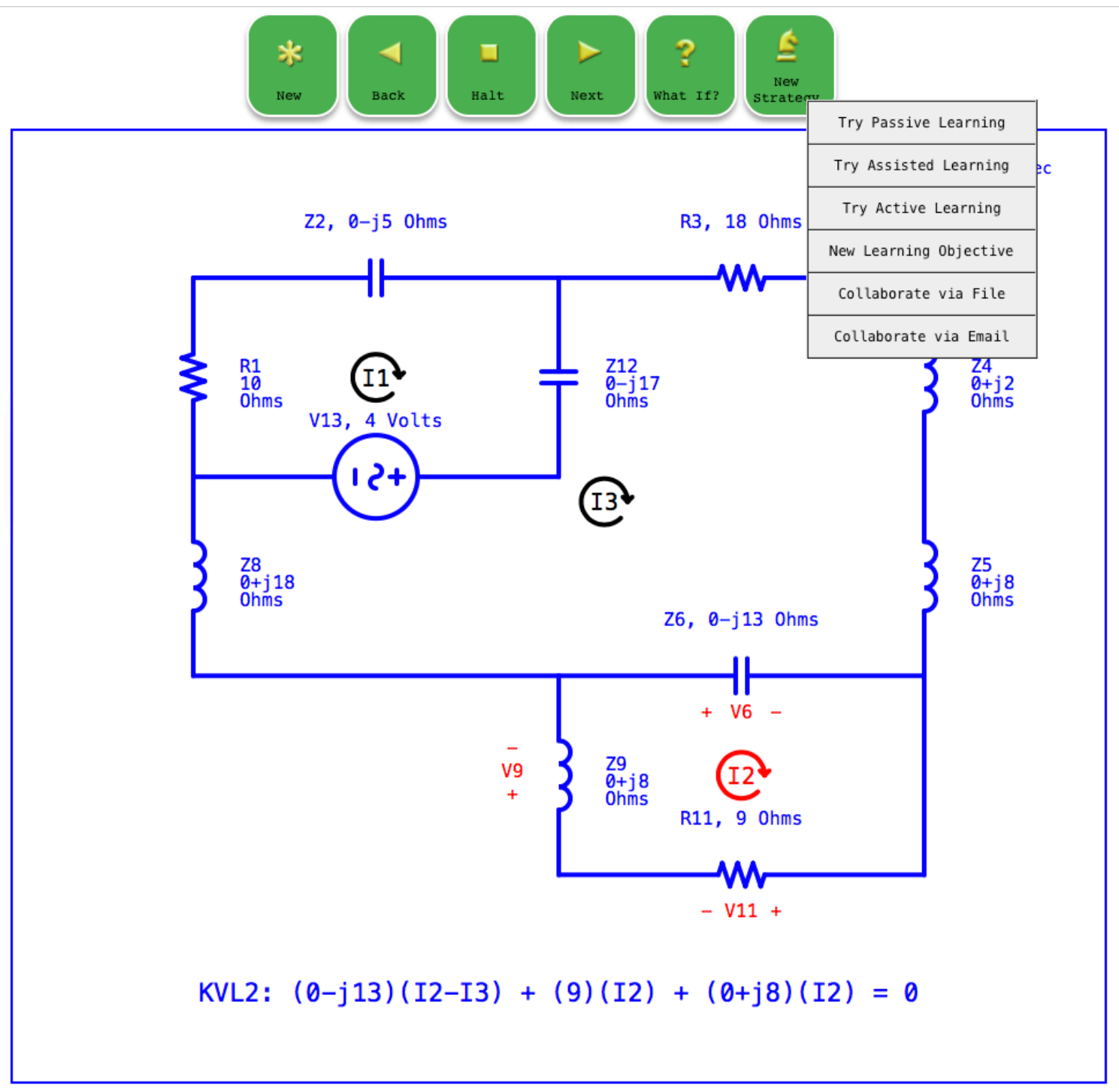

Fig 5. Students may elect to change their learning strategy as desired. For example during a passive analysis, they may switch to an assisted or active approach. They can also change learning objective and try an alternate method of analysis (mesh, nodal or equivalent circuits). These features promote interleaved approaches to learning.

Using CATE, students can study individual learning objectives using various modes such as passive, assisted or active. They can also interleave these different opportunities, for example switching between passive and assisted modes. Or they can examine a given circuit from the perspective of a difference analysis technique (switch from nodal to mesh for example). This allows students to build directly on prior abilities using a scaffolding type of approach and can help make connections between different techniques. See Figure 5. Scaffolding also helps students succeed in situations that may be unfamiliar or too challenging given their current abilities [9]. 

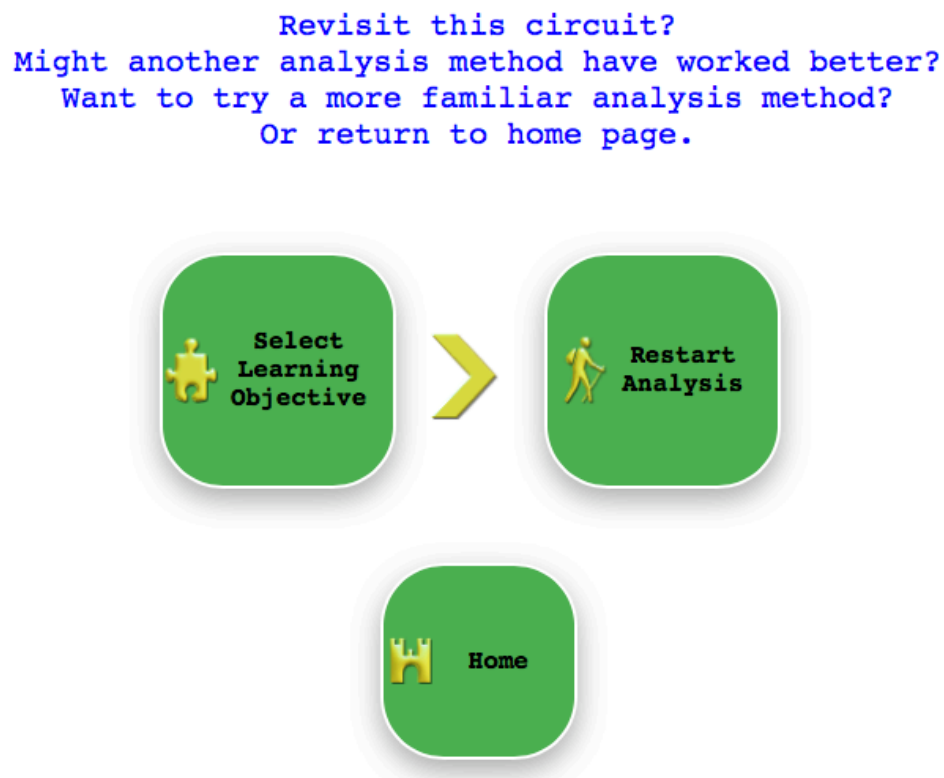

Fig 6. Following passive or assisted mode, students are encouraged to reflect on the prior analysis for comparative strengths of the method. They can select a new analysis method and then revisit the same circuit (e.g. switch from nodal to mesh). These features promote reflection, scaffolding and interleaved learning.

In addition to scaffolding that can help develop abilities, CATE also encourages students to interleave their learning efforts. Numerous studies have shown that interleaving different types of problems, different procedures and varied specific situations all helped student performance in both learning and retention. Benefits of an interleaved approach have been reported in a wide variety of learning domains, including verbal recall [4], medical students [5], solving anagrams [6], art appreciation [7] and solving math problems [8].

When students end a session of passive or assisted learning they are presented with options shown in Figure 6. Students are encouraged to reflect on alternate methods of analysis for the prior circuit. This branch point in a student's use of CATE provides an opportunity for reflection, scaffolding and interleaved learning.

\section{Assessment Methodology and Design of Embedded Tools}

CATE is designed to allow students to navigate through different features, as driven by their interest and need. This can be a circuitous path unlike the sequential nature of a lecture video, for example. The non-sequential and self-directed usage also presents a challenge for assessment. For example we can't simply time how long a student watches a video. Which features/modes appear the most useful? Which sequencing between features may yield the most benefit? Could a style of use be recommended for a particular student? We need embedded assessment tools that can support a wide range of questions. 
Regarding our assessment methodology, we desire an approach where using CATE is optional. Hence a quasi-experimental approach is appropriate. This will allow students to use CATE to varying degrees and in self-selected ways. This differs from a true experiment where one group of students would use CATE and another group would not. Potential quasi-independent variables include time on task, as measured by mouse clicks for example, or the percentage of usage in assisted mode versus passive.

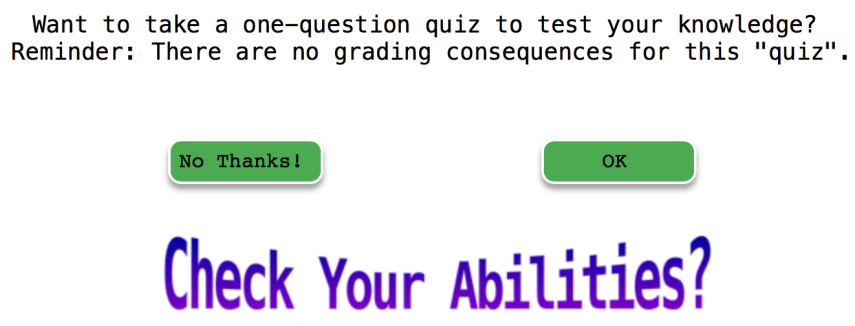

The last time you took one of these quizzes was over 24 hours ago.

A 10 minute countdown timer starts when you click OK - Get Ready!

Fig 7. Prompt offering students to take an internally generated quiz, to augment assessment data.

Potential dependent variables for our study include the percentage of correctly answered active learning questions, or the number of mouse clicks used to define an equation in assisted learning mode. To increase our opportunities to assess student abilities we have introduced additional internal quiz questions that pop up every 10-20 minutes during the course of normal usage. See Figures 7 and 8. Internal quiz questions are optional. When students attempt one of the internal quiz questions, other features of CATE are locked out; the student may only respond to that question. This was done to get a more accurate assessment of student abilities.

To further expand the opportunities for assessment we provide a (CSV) file upload feature that allows instructors to import grades from a class quiz or similar source. The instructor also specifies when the external assessment took place so that a complete time sequence can be maintained for each student.

Because students use CATE at their own pace and at times of their own choosing, we don't have a clear opportunity for a single pre-test and post-test. We have designed the longitudinal tracking system with the objective of performing a time series analysis. Our time series include activity on CATE interspersed by assessment data (internal to CATE or imported from an external source) as shown in Figures 9 and 10. A positive impact would be seen as a tendency for better performance following more activity with CATE. The slope of these upward trends will vary with individual users. We are curious how the trends may also vary based on particular styles of usage of CATE (e.g. active, assisted or passive). We do not envision our use of a time 
series to be as clearly delineated as those in medical studies [13] where a single treatment or policy change interrupts a time series of measurements in a precise fashion. Rather our time sequences will be interrupted in a more random manner. Nevertheless we have designed our assessment tools to track styles of usage (e.g. assisted versus passive learning) with the hope of observing results similar to Skromme [10-12].

What is the current thru R3?

\begin{tabular}{llll}
\hline Your Answer: & Timer: $8: 20 \quad$ Submit & Skip \\
\hline
\end{tabular}

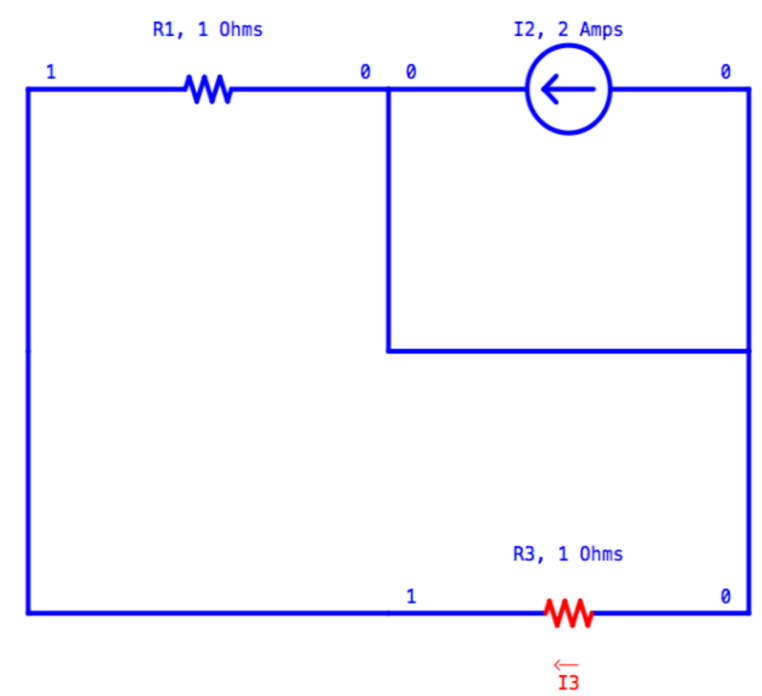

Fig 8. Example of a question from an internally-generated quiz. Student response times are stored along with correct/incorrect results from automatic grading.

\begin{tabular}{|c||c|c|c||}
\hline \hline & Activity & TS & DS \\
\hline \hline ly.edu & EQ|DC|1|BEGIN.ACTV & 1484200316 & $\begin{array}{c}2017-01-12 \\
00: 51: 56\end{array}$ \\
\hline \hline ly.edu & MESH|DC|1|NEXT.ACTV & 1484200389 & $\begin{array}{c}2017-01-12 \\
00: 53: 09\end{array}$ \\
\hline \hline ly.edu & MESH|DC|1|NEXT.ACTV & 1484200394 & $\begin{array}{c}2017-01-12 \\
00: 53: 14\end{array}$ \\
\hline \hline ly.edu & MESH|DC|1|NEXT.ACTV & 1484200397 & $\begin{array}{c}2017-01-12 \\
00: 53: 17\end{array}$ \\
\hline \hline ly.edu & MESH|DC|1|NEXT.ACTV & 1484200399 & $\begin{array}{c}2017-01-12 \\
00: 53: 19\end{array}$ \\
\hline ly.edu & MESH|DC|1|NEXT.ACTV & 1484200403 & $\begin{array}{c}2017-01-12 \\
00: 53: 23\end{array}$
\end{tabular}

Fig 9. Detailed version of longitudinal tracking data. Individual mouse clicks are captured and time stamped. Encoding for student activities includes learning objective, $A C$ vs $D C$, difficulty level, as well as learning strategy (e.g. active) 
Our system provides variable levels of detail as shown in Figures 9 and 10. Individual mouse clicks are presented in Figure 9, versus a run-length encoded version in Figure 10. Our collected data can also be used to examine correlations between the total time on task and performance, simply by ignoring the time sequencing information and then accumulating data across all users. We have intentionally designed variable levels of detail in order to maintain flexibility.

\begin{tabular}{|c|c|c|c|}
\hline & RLC & $\mathbf{N}$ & Start \\
\hline ly.edu & EQ|DC|1|ACTV & 1 & 2017-01-12 00:51:56 \\
\hline ly.edu & MESH|DC|1|ACTV & 5 & 2017-01-12 00:53:09 \\
\hline ly.edu & $\mathrm{KCL}|\mathrm{DC}| 3 \mid \mathrm{ACTV}$ & 1 & 2017-01-17 15:23:52 \\
\hline ly.edu & MESH|DC|3|ALQUIZ_ACK.ASMT & \begin{tabular}{|l|}
1 \\
\end{tabular} & 2017-01-17 15:25:40 \\
\hline ly.edu & MESH|DC|3|ACTV & \begin{tabular}{|l|}
1 \\
\end{tabular} & 2017-01-17 15:26:37 \\
\hline y.edu & NODAL|DC|3|ACTV & 1 & 2017-01-17 15:32:47 \\
\hline sy.edu & THEV_NORT|DC|3|ASST & 1 & 2017-01-12 10:19:49 \\
\hline ly.edu & MESH|DC|3|ASST & 63 & 2017-01-12 10:23:23 \\
\hline sy.edu & MESH|DC|2|ASST & 7 & 2017-01-12 10:38:07 \\
\hline sy.edu & THEV_NORT|DC|3|ASST & 1 & 2017-01-16 18:27:14 \\
\hline sy.edu & MESH|DC|3|ASST & 38 & 2017-01-16 18:27:34 \\
\hline
\end{tabular}

Fig 10. Run-length encoded version of longitudinal tracking data.

The availability of tracking data for usage studies provides an opportunity to present usage information across student population. This allows an instructor to see which learning objectives and at what difficulty level. See Figure 11. This example demonstrates a sampling of usage by course learning objective.

\section{Conclusion and Future Efforts}

We see the potential for CATE to compliment the learning resources of a traditional introductory circuits course. We have introduced it for purposes of reviewing material from a prior course, and as a means to help introduce new material.

Previous student opinion surveys on CATE [15] were favorable. Our next major step will be to use the assessment tools described herein to more critically evaluate the benefits of CATE with larger numbers of students. It has been interesting to review the limited usage data we have captured thus far. Some students who were using CATE to review a prior course took advantage of a wide range of learning objectives and learning strategies. In another instance students were reviewing for a midterm exam and were much more focused in their usage. We draw no conclusion from these observations but we are pleased to see diverse usage of the system by different students in different settings.

Moving forward we are interested in using CATE to generate homework and quizzes. These problems can be distributed by email and automatically graded. Problems can also include topological and numerical variations rather than being 
the same for all students. We are hoping to encourage on-going usage with CATE as opposed to cramming with CATE prior to an exam.

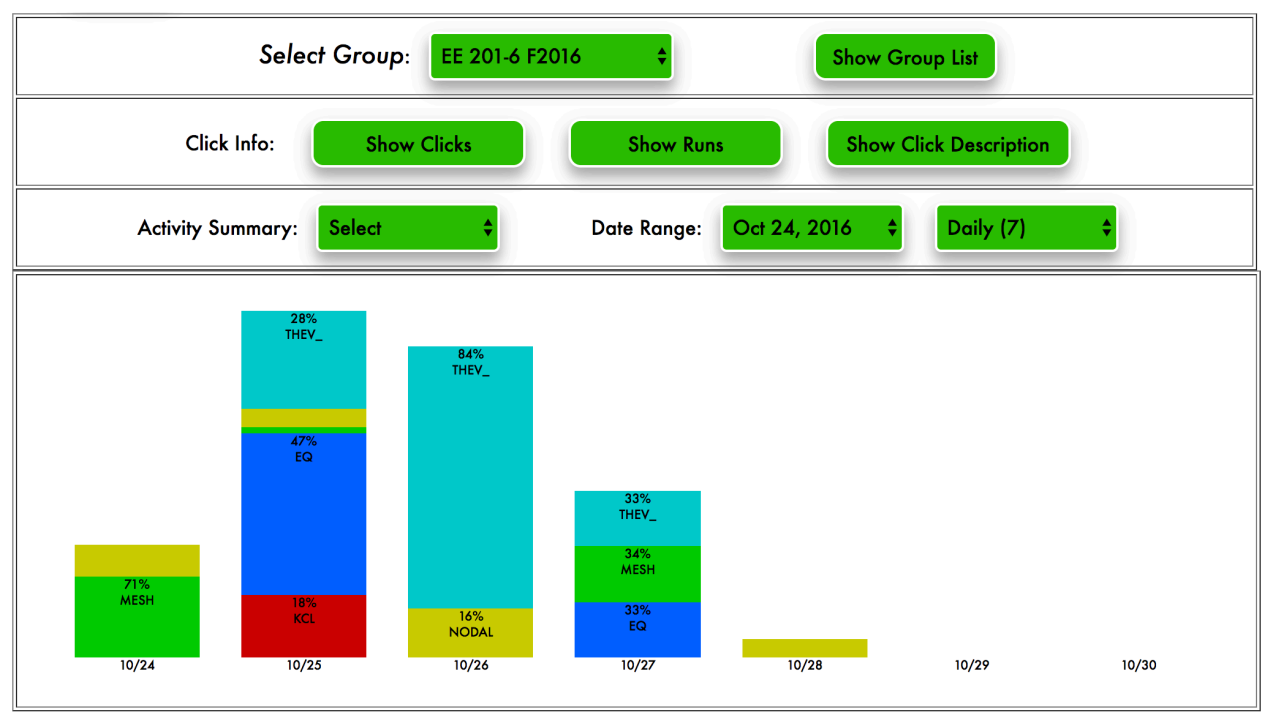

Fig 11. Example of cross-sectional data presenting usage activity for students in a class, as a function of time. Learning objectives appear in different colors and the time scale can be adjusted.

\section{References}

[1] Roediger and Karpicke, The power of testing memory: Basic research and implications for education practice, Perspectives on Psychological Science 1 (2006), 181-210.

[2] Arnold and McDermott, Test-potentiated learning: Distinguishing between direct and indirect effects of tests, Journal of Experimental Psychology: Learning, Memory and Cognition 39 (2013), 940-945.

[3] Brown, Roediger and McDaniel, Make It Stick, Harvard University Press (2014).

[4] Cepeda, et al., Distributed practice in verbal recall tasks; A review and quantitative synthesis, Psychological Bulletin 132 (2006), 354-380.

[5] Moulton, et al., Teaching surgical skills: What kind of practice makes perfect? Annals of Surgery 244 (2006), 400-409.

[6] Goode, Geraci and Roediger, Superiority of variable to repeated practice in transfer on anagram solution, Psychonomic Bulletin \& Review 15 (2008), 662-666.

[7] Kornell and Bjork, Learning concepts and categories: Is spacing the "enemy of induction"?, Psychological Science 19 (2008), 585-592.

[8] Rohrer and Taylor, The shuffling of mathematics problems improves learning, Instructional Science 35 (2007), 481-498.

[9] Quintata, et al., A Scaffolding Design Framework for Software to Support Science Inquiry, Journal of the Learning Sciences 13 (3) (2004), 337-386.

[10] C. D. Whitlatch, Q. Wang, and B. J. Skromme, "Automated problem and solution generation software for computer-aided instruction in elementary linear circuit analysis," in Proceedings of the 2012 ASEE Annual Conference \& Exposition. Washington, D.C., ASEE, (2012).

[11] B. J. Skromme et al., "Teaching linear circuit analysis techniques with computers," in Proceedings of the 2013 ASEE Annual Conference \& Exposition, Atlanta, GA, ASEE, (2013).

[12] Skromme, et al., Computer-aided instruction for introductory linear circuit analysis, Frontiers in Education Conference El Paso, TX, (2015).

[13] A. K. Wagner, S. B. Soumerai, F. Zhang and D. Ross-Degnan, Segmented regression analysis of interrupted time series studies in medication use research, Journal of Clinical Pharmacy and Therapeutics (2002) 27, 299-309 
[14] F. W. DePiero, "Work-in-Progress: Design of an online learning coach," in Proceedings of the 2013 ASEE Annual Conference \& Exposition, Atlanta, GA, ASEE, (2013).

[15] F. W. DePiero et al., "CATE: A Circuit Analysis Tool for Education," in Proceedings of the 2016 ASEE Annual Conference \& Exposition, New Orleans, LA, ASEE, (2016).

[16] Evaluation of Evidence-Based Practices in Online Learning: A Meta-Analysis and Review of Online Learning Studies, U.S. Department of Education, Office of Planning, Evaluation, and Policy Development, Washington, D.C., (2010).

[17] LTSpice, http://www.linear.com/designtools/software/

[18] Circuits.IO, https://circuits.io/

[19] Mastering by Pearson, https://www.pearsonmylabandmastering.com 